\title{
Influencia humana en las fluctuaciones poblacionales de erizos de mar
}

\author{
José Carlos Hernández \\ Ecología de Comunidades Marinas y Cambio Climático. Departamento de Biología Animal, Edafología y Geología. \\ Universidad de La Laguna; Tenerife. Islas Canarias. jocarher@ull.es
}

Recibido 31-I-2017. Corregido 04-III-2017. Aceptado 23-VI-2017.

\begin{abstract}
Human influence on sea urchin population fluctuations. Echinoids play an important role in marine ecosystems structuring. Often, their population density experience markedly fluctuations that promote a state shift in the ecosystems they inhabit. Population increments of some sea urchins may cause catastrophic changes in temperate areas of the planet by decimating the erect macroalgae cover. These population increments results in unproductive and very stable assemblages, known as "blanquizales" (ericeras/moradales), or sea urchin barren ground. Macroalgae are the main ecosystem engineers in temperate areas and generate a suitable nursery and feeding habitat for fishes. These algae stands are also important zones for biofiltration of coastal waters and $\mathrm{CO}_{2}$ uptake. The main consequence of vegetated biomass lost is a trophic disequilibrium that generates important economic losses for artisanal fisheries and tourism. In tropical areas, sea urchin's outbreaks can cause bioerosion in coral reefs. However, the most important event to highlight was the mass mortality occurred in the Caribbean during the 80 's. This event favored the development of algae communities that suffocated the coral reef ecosystem. Therefore, both in temperate and tropical areas of the planet, these boom-bust echinoids generate undesired ecosystems states. Very recently, various global scale collaborative papers have highlighted a clear anthropogenic cue. Human activity though overfishing or favoring global warming, weakens marine ecosystem resilience and promote these catastrophic ecosystem shifts. To mitigate the effects of these population changes different management strategies have been used. For instance, in temperate areas, sea urchin reduction actions (manually or by using quick lime), sea urchin harvesting or the implementation of marine reserves have been used, with contrasting results. In Caribbean coral reefs affected by urchin mass mortality, some sea urchin juvenile's reintroduction plans have been used but with very low effectiveness. The more feasible ecosystem scale strategy due to its preventive nature, seems to be the implementation of protected areas. However, the impact of global warming will exceed our capacity to manage marine ecosystems locally and will required more efficient global actions to prevent undesired sea urchin fluctuations. Rev. Biol. Trop. 65(Suppl. 1): S23-S34. Epub 2017 November 01 .
\end{abstract}

Key words: sea urchins; population dynamics; community ecology; climate change; predation; marine reserves.

Erizo de mar, marino, con cien lanzas afiladas que a todas partes apuntan a veces son cien espadas dispuestas a la pelea, que se separan o juntan

al ritmo de la marea
Erizo de mar, marino, una flor toda puñales, que ajena a los arenales ancló en la roca el destino sólo Dios sabe tu sino, sólo él tiene la clave, lancero fino en su enclave... erizo de mar, marino.

Domingo Chico González Sueños de Amor y de Mar Poeta Canario 
En muchas regiones del océano, desde los ecosistemas templados a tropicales, los erizos de mar son una pieza clave, ya que son capaces de alterar el hábitat donde viven, tanto por sus elevadas abundancias como por su ausencia (Lawrence, 1975). Podemos enumerar al menos 12 especies que han sido señaladas como causantes de este tipo de modificaciones de hábitat, entre ellas las especies más destacables por su capacidad para modificar el hábitat están dentro de las Familias Arbaciidae, Diadematidae y Strongylocentrotidae (Uthicke et al., 2009; Filbee-Dexter \& Scheibling, 2014; Ling et al., 2015). Estas especies poseen diferentes hábitos alimenticios, que van desde omnívoros/ carnívoros como Arbacia, a herbívoros y detritívoros como sería el caso de los Diademátidos o Strongylocentrotus (Lawrence, 1975; Hernández et al., 2008; Wangensteen et al., 2011). $\mathrm{Si}$ bien, todos presentan una característica común, desarrollan larvas planctotróficas de vida libre. Esta característica biológica puede, en parte, ayudar a explicar el porqué de estas fluctuaciones poblacionales, pues este tipo de larvas tienen una estrategia de éxito o fracaso, ya que están sujetas al efecto "Allee" (Uthicke et al., 2009) (Fig. 1).

Para visualizar mejor las consecuencias de sus fluctuaciones poblacionales, nos bastaría con imaginar cómo un bosque de kelp exuberante y lleno de vida, se convierte en un desierto marino (Fig. 2a). Al aumentar las densidades de erizos, la estructura trófica se derrumba debido a la pérdida de biomasa vegetal, produciendo numerosos efectos indirectos que llegan en último término a afectar a las flotas pesqueras y la sociedad que vive de los recursos del mar. Estas fluctuaciones poblacionales también afectan a los ecosistemas arrecifales y pueden llegar a bioerosionar los arrecifes, modificando su estructura y comunidades asociadas que buscan en ella refugio y alimento (Fig. 2b). Esto se ha observado en zonas del Pacífico pero también en el Caribe, con anterioridad a la mortalidad ocurrida en los años 80 (Lessios, 1988). Ésta mortalidad masiva de erizos también generó un desequilibrio trófico, ya que los erizos eran los herbívoros más importantes de estos sistemas

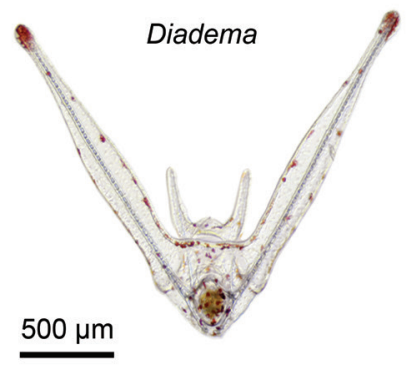

Arbacia

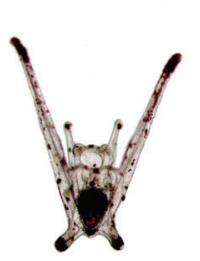

\section{Strongylocentrotus}

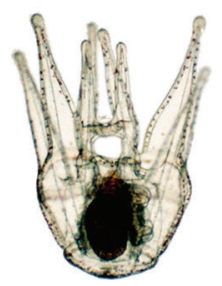



Fig. 1. Larvas planctotróficas de las especies Diadema africanum, Arbacia lixula y Strongylocentrotus purpuratus, equinoideos capaces de formar extensos blanquizales. La foto de A.lixula fue cedida por O.Wangensteen y la de S.purpuratus por A.Cameron.

Fig. 1. Planktotophic larvae of the species Diadema africanum, Arbacia lixula and Strongylocentrotus purpuratus, echinoids capable of creating extensive barrens. Picture of Arbacia larvae courtesy of O. Wangensteen and Strongylocentrotus courtesy of A.Cameron. 

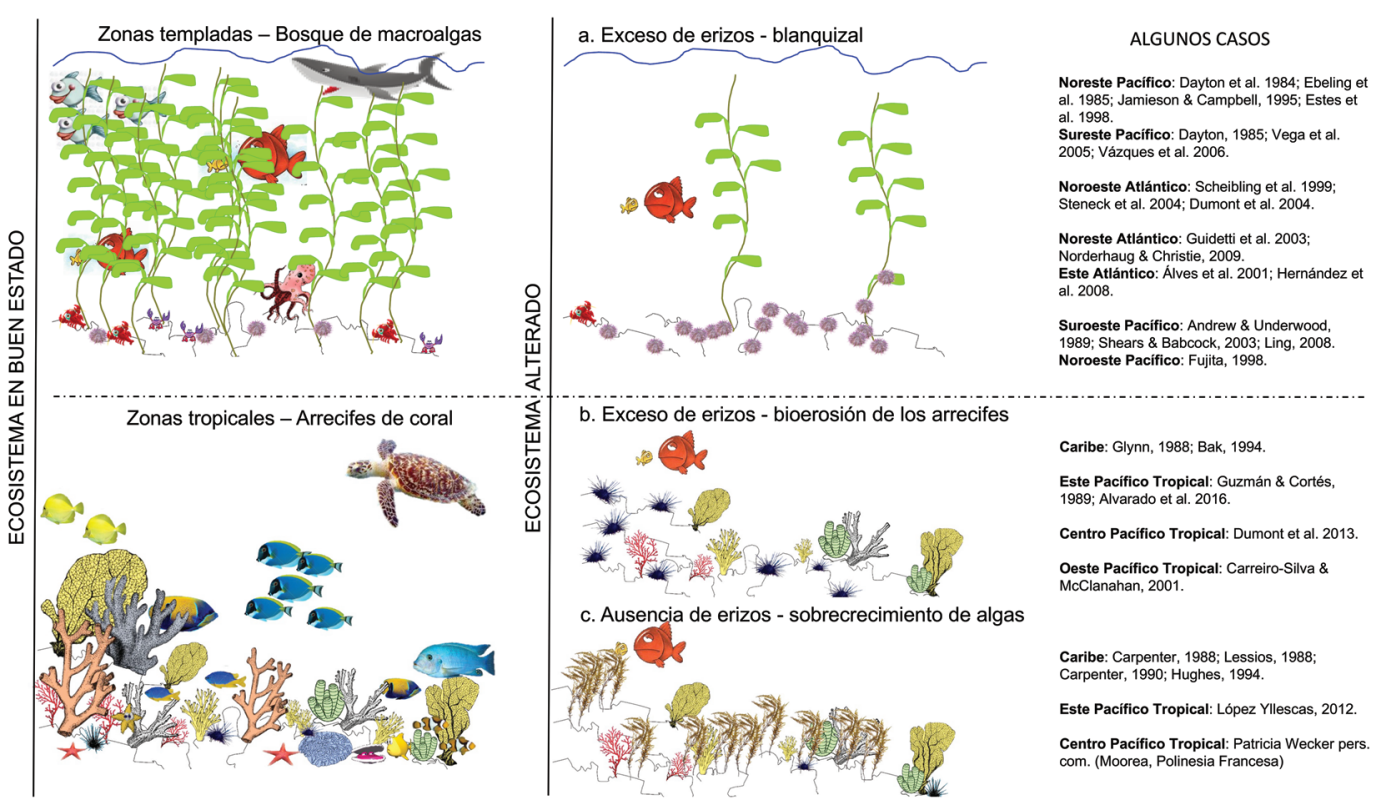

Fig. 2. Estados alternativos de los ecosistemas marinos costeros de zonas templadas y tropicales. En el lado izquierdo del esquema se puede ver un estado conservado y en el lado derecho los estados alterados debidos a las fluctuaciones en la densidad poblacional de erizos. a. Blanquizal originado por la alta densidad de erizos de mar que reducen drásticamente la cobertura de macroalgas. b. Bioerosión producida por el exceso de erizos que consume y destruye la estructura coralina. c. Mortalidad masiva de erizos que beneficia el crecimiento excesivo de macroalgas capaces de asfixiar a los arrecifes, impidiendo además el asentamiento de nuevas colonias de corales.

Fig. 2. Alternative states of the coastal marine ecosystems from temperate and tropical areas. On the left-hand side of the scheme, it can be observed a conserved state and in the right-hand side, the alternate state due to fluctuations on sea urchin population density. a. Barren created by the high urchin density that drastically reduce the algae cover. b. Bioerosion due to sea urchin excess that consume and destroy the coralline structure. c. Sea urchin mass mortality that benefice the overgrowth of macroalgae capable of suffocate the reefs, avoiding the settlement of new corals colonies.

y su pérdida lleva afectando unos 30 años a la ecología de los arrecifes, por ejemplo por el crecimiento excesivo de algas como Sargassum y otras (Lessios, 2016) (Fig. 2b). Así pues, tanto por exceso, como por defecto, las poblaciones de equinoideos juegan un papel clave en varios sistemas marinos del planeta.

Este papel tan importante de los erizos de mar ha tenido también relevancia social y en los años noventa, por ejemplo, fue portada del New York Times Science (William, 1999) con un reportaje sobre el artículo de J. Estes et al. (Estes et al., 1998). Este artículo describía el efecto de la desaparición de las nutrias por depredación de las orcas que a su vez generó una expansión de los blanquizales en Alaska, islas Aleutianas y algunas zonas de California. Éste fue uno de los casos más llamativos porque el trabajo de Estes et al., (1998) ligaba la desaparición de los bosques de kelp con la reducción en la población de nutrias debido a la depredación por Orcas. Aunque, lo más interesante de todo es que esta serie de interacciones habían sido originadas por los humanos. Es decir, las Orcas habían cambiado su dieta y habían empezado a comer nutrias porque las poblaciones de sus presas normales, focas y leones marinos, se habían reducido drásticamente debido a la sobrepesca de los stocks de peces por parte de los humanos. Así, este fue uno de los primeros ejemplos que demostraba la influencia humana en las fluctuaciones poblacionales de erizos de mar.

En las zonas templadas del planeta existen numerosos casos de fluctuaciones poblacionales de erizos de mar, tanto de fenómenos 
de asentamientos masivos devastadores para los bosques de algas, como grandes mortandades que han favorecido el restablecimiento de los sistemas algales (Filbee-Dexter \& Scheibling, 2014). Estos fenómenos de alternancia en los ecosistemas costeros han llamado nuestra atención desde hace tiempo, dada la magnitud de los efectos que pueden acarrear. Uno de los primeros en darse cuenta de esto y de hacerse las primeras preguntas fue, J.M. Lawrence (1975). Hoy en día, las preguntas de Lawrence siguen estando vigentes dados los casos actuales de fluctuaciones poblacional de erizos en las costas de Tasmania, Japón o las islas Canarias (Ling et al., 2015). Por este motivo, creímos fundamental organizar en 2012 un Workshop Internacional para tratar de responder a estas preguntas (ULL-Media 2014). Como resultados de esta reunión que llevamos a cabo en la Universidad de La Laguna (Tenerife, islas Canarias), se elaboró un libro que recoge los casos mejor estudiados hasta el momento (Palacios et al., 2013). Recientemente, además, hemos publicado un trabajo global sobre las dinámicas de cambio entre estados algales y blanquizales, extremadamente útil para las estrategias de manejo y conservación de los ecosistemas marinos de fondos rocosos someros (Ling et al., 2015).

\section{¿Qué hemos aprendido estudiando los blanquizales generados por el erizo Diadema africanum?}

En Tenerife, actualmente, hasta un $64.59 \%$ de los fondos rocosos están ocupados por este erizo de mar (Figuras 3-4). Cuando las densidades están por encima de 4 ind $\mathrm{m}^{-2}$, es capaz de eliminar toda la cubierta vegetal y adueñarse del sistema (Hernández et al., 2008), trabajos posteriores nos mostraron cómo también esa reducción en la cobertura de algas hace que disminuya la riqueza y diversidad de los sistemas (Sangil et al., 2014), que se empobrecen al aumentar la densidad de erizos, también los peces, recursos pesqueros como la vieja (Sparisoma cretense) casi desaparece por completo en las zonas de blanquizal, tanto los adultos como los juveniles (Clemente et al., 2009).

Conociendo sus efectos comenzamos a experimentar con estrategias de reducción de las densidades en zonas controladas, con el objetivo

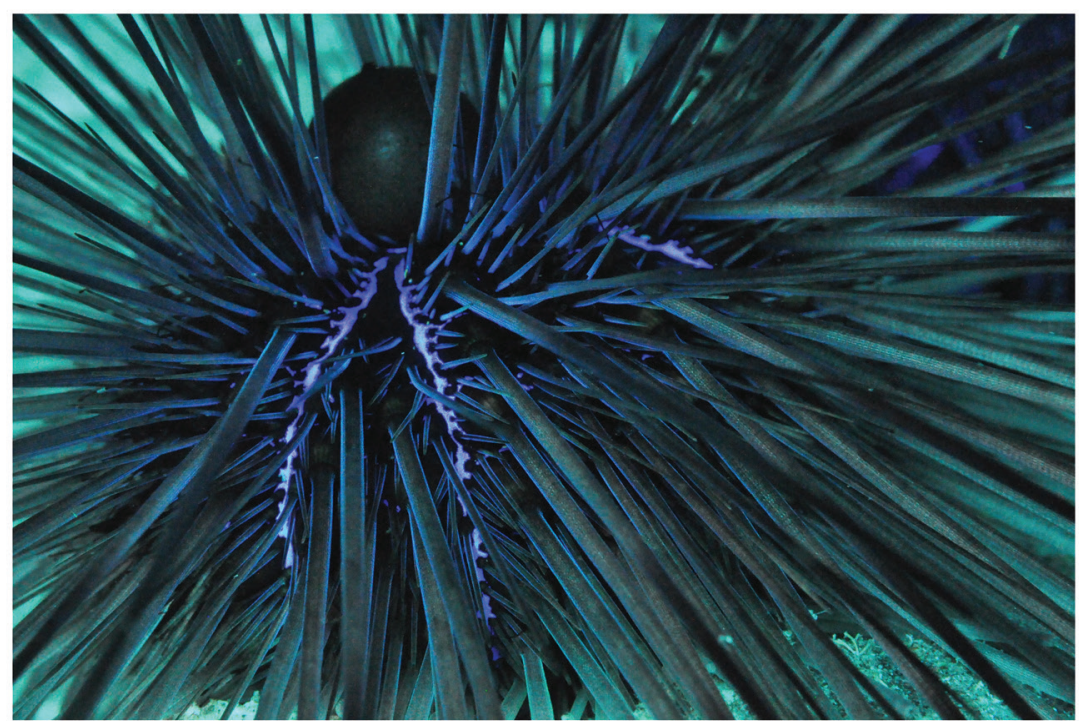

Fig. 3. Erizo de mar Diadema africanum, común en los archipiélagos de Madeira, Salvajes e islas Canarias. Se distribuye en el Atlántico Este desde las islas Azores hasta el Golfo de Guinea e islas de Cabo Verde.

Fig. 3. Sea urchin Diadema africanum, commonly found in the archipelagos of Madeira, Salvajes and the Canary Islands. It is distributed in the East Atlantic from Azores Islands to Guinean Gulf and Cabo Verde Islands. 


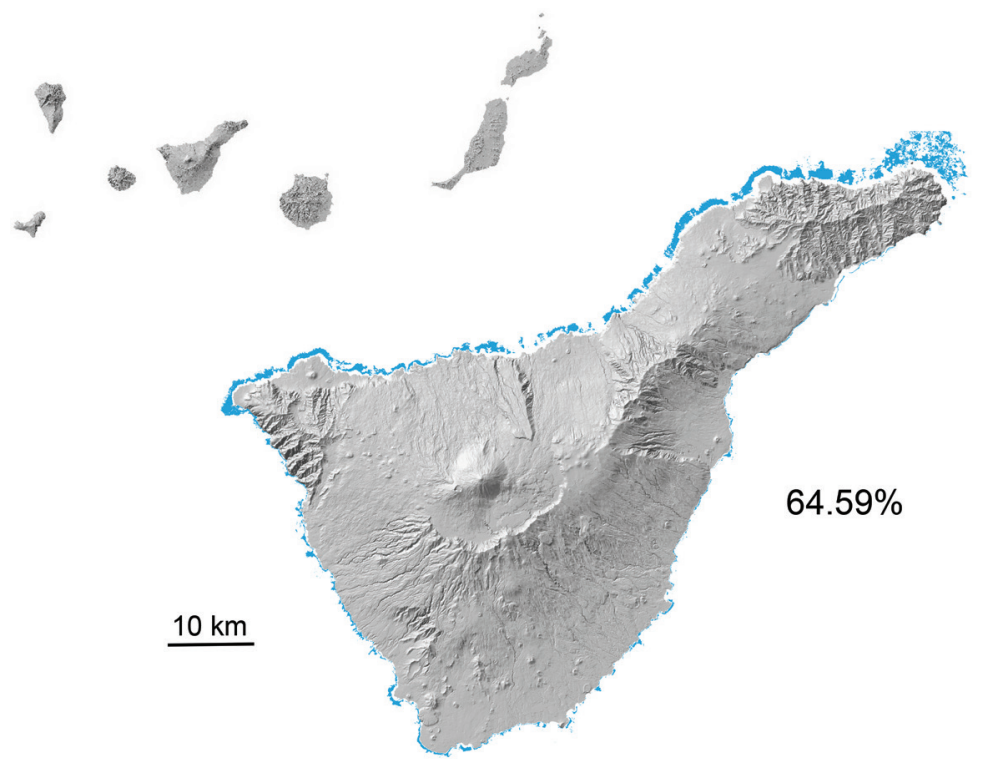

Fig. 4. Mapa del archipiélago Canario y ampliación de la isla de Tenerife, señalando la extensión de blanquizal que rodea a la isla.

Fig. 4. Map of the Canarian Arquipelago and an amplification of Tenerife Island, highlighting the barren extension around the island.

de recuperar el sistema algal (Falcón et al., 2004). Si bien, la recuperación era rápida cuando se eliminaba la totalidad de los erizos de un área determinada, la presencia de adultos en los alrededores no dejaba que la zona se mantuviera estable, puesto que desde que cesaba la acción de eliminación los erizos volvían a dominar el sistema (Hernández et al., in prep). Por lo que no parece una solución muy estable.

En relación a lo anterior, estudios recientes a nivel global nos han mostrado el que existe una histéresis en la dinámica de alternancia entre comunidades, por lo que para poder recuperar las coberturas algales prístinas hay que reducir a cero las densidades de erizos, si no, la recuperación no ocurre (Ling et al., 2015) (Fig. 5). Esto también quiere decir que muy pocos erizos son capaces de mantener un blanquizal una vez formado. Ambos estados del ecosistema tienen una serie de procesos de retroalimentación positiva que los hacen muy estables en el tiempo, por lo que una vez formado el blanquizal es muy difícil volver atrás (Filbee-Dexter \& Scheibling, 2014). Así que más vale prevenir que curar. Este fracaso hizo que nos hiciéramos más preguntas que recientemente hemos empezado a resolver.

- ¿Desde cuándo aparecen estos blanquizales?

Uno de los primeros en recolectar Diade$m a$ en las islas Canarias fue Ole Theodor Mortensen durante los años 30 (Mortensen, 1943). Veinte años después, Johnston publica una comunicación a un congreso donde describe que en las costas de Lanzarote aparecen vastas poblaciones del erizo Diadema, que él confunde con Centrostephanus longispinus (Johnston, 1969). Desde los años 30 aparecen registros claros de la presencia de Diadema en Canarias y posiblemente en grandes densidades desde los años 60. Por lo que los blanquizales están presentes en las Islas desde hace 50 años como mínimo (Hernández et al., 2013). 


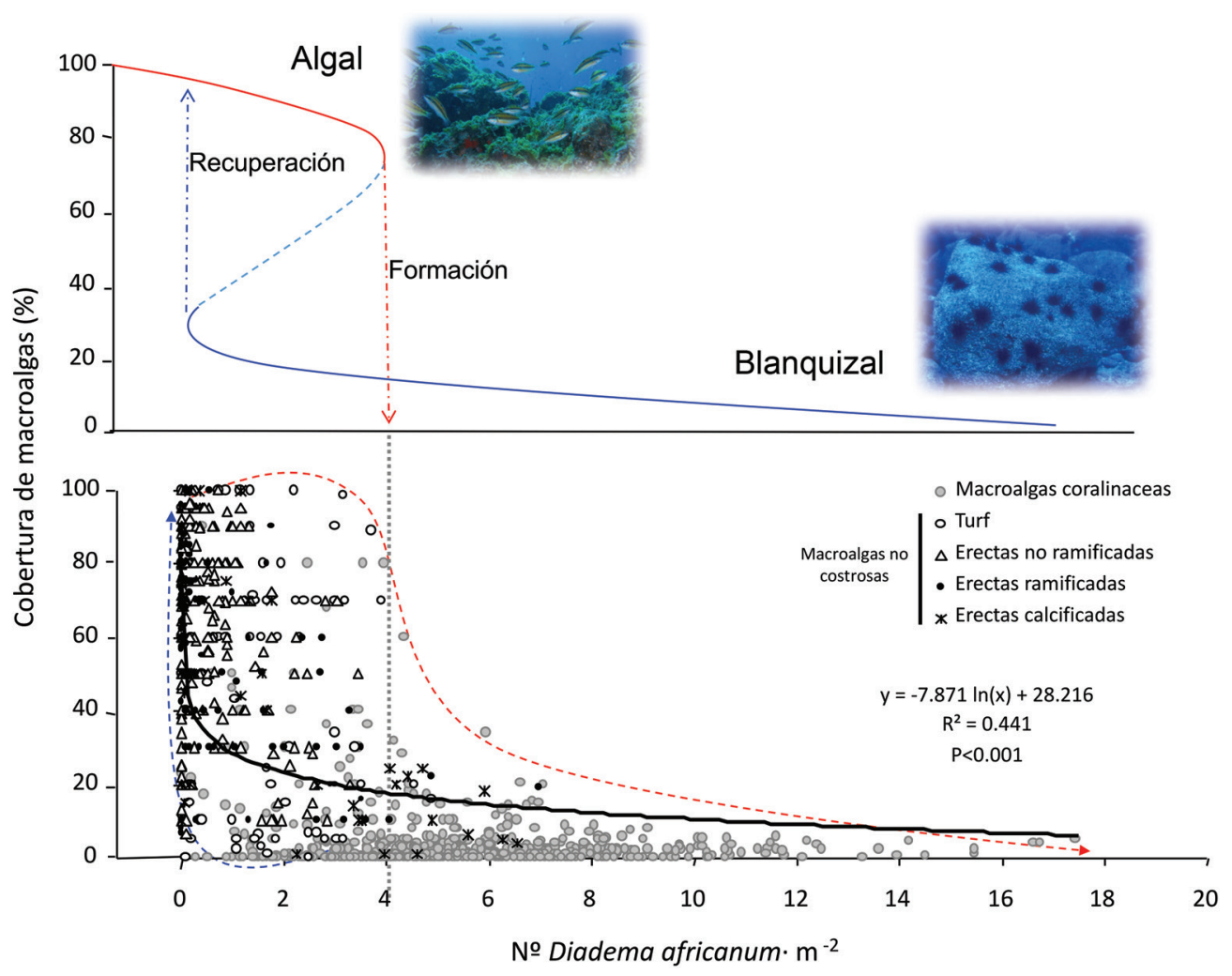

Fig. 5. Representación del cambio de estado catastrófico que ocurre en las comunidades de macroalgas de las islas Canarias cuando los erizos alcanzan una densidad de $4 \mathrm{ind} \cdot \mathrm{m}^{2}$. Estas densidades generan blanquizales estables en el tiempo y cuya reversión es muy complicada. La recuperación de los fondos de macroalgas sólo se logra cuando las densidades de erizos son inferiores a $1 \mathrm{ind} \cdot \mathrm{m}^{2}$.

Fig. 5. Representation of the catastrophic phase shift that occurs on the macroalgae assemblages of the Canary Islands when the sea urchins rich 4 ind $\cdot \mathrm{m}^{2}$. These densities generate stable barrens in time, which have a complicate reverse shift. The recovery of macroalgae state only occurs with sea urchin's density under $1 \mathrm{ind} \cdot \mathrm{m}^{2}$.

- ¿Cuándo llegó este equinoideo a las islas Canarias?

Gracias a los análisis realizados por el investigador Harilaos Lessios y su equipo, sobre la filogenia del género, podemos saber que hace 2 Ma se separa un clado antillano de otro puramente Atlántico (Lessios et al., 2001). Este clado atlántico lo hemos descrito recientemente y lo hemos denominado Diadema africanum, por su presencia exclusiva en la placa africana (Rodríguez et al., 2013). El estudio de la historia demográfica también nos ha permitido conocer que hace unos 80000-125000 años atrás ocurrió un aumento de la tasa de mutaciones y por ende de las densidades poblacionales de este equinoideo en el Atlántico Este (Lessios et al., 2001). En esa época, las islas Canarias tenían un clima más cálido que el actual. En términos geológicos, ese periodo es conocido como el periodo isotópico $5 \mathrm{e}$, y coincide con un periodo cálido en el que los yacimientos indican la presencia de la especie de gasterópodo Strombus y de corales como Siderastrea, de hábitos más tropicales (Carracedo et al., 2005). Para hacernos una idea, a una escala más humana, en esa época el Homo florensis andaba por indonesia, el Homo nearthentalensis por Europa y nosotros no habíamos salido de África. Así que, sin duda alguna, el Diadema llegó a Canarias mucho antes que el hombre. 
- ¿Se han registrado fluctuaciones de sus poblaciones?

A pesar de que la presencia de la especie es anterior a la llegada del hombre y muchas fluctuaciones pudieron ocurrir en ese entonces, sí hemos sido testigos de oscilaciones en las poblaciones de erizos. Por ejemplo, en los años 90 pudimos registrar como en tan solo diez años los erizos llegaban al islote de Montaña Clara y dominaban por completo el paisaje (1995-2005) (Hernández et al., 2008). En los últimos años, también hemos sido testigos de una mortalidad masiva de este equinoideo que ha favorecido la recuperación de las coberturas de macroalgas en algunas localidades (Clemente et al., 2014). La recuperación o no de las comunidades de macroalgas dependió de la densidad inicial de erizos en cada localidad. Por ejemplo, en zonas con alta densidad la mortalidad no consiguió revertir la situación de blanquizal, sin embargo, muchas localidades con densidades por debajo de los 4 ind $\mathrm{m}^{-2}$ han reflorecido (Sangil \& Hernández, in prep).

- ¿Qué factores están relacionados con la densidad de equinoideos?

En Canarias existe un patrón de distribución de las poblaciones de erizos relacionado con la orientación frente al oleaje pero también relacionada con la actividad turística, pesquera, es decir, con el número de habitantes por perímetro de costa. Siendo las islas más explotadas aquellas que presentan una mayor densidad de erizos (Hernández et al., 2008). Sin embargo, esto no es tan sencillo como parece y existen muchos factores que regulan las poblaciones o pueden influir en su fluctuación: desde el aporte larvario a la presencia de depredadores. Y además, estos factores fluctúan espacial y estacionalmente por lo que el panorama es más intrincado aún. Así que tenemos que empezar por el principio, las larvas.

El estudio del periodo planctónico de esta especie ha resultado ser muy interesante y nos ha permitido descubrir, por ejemplo, que las altas temperaturas benefician la supervivencia, favoreciendo asentamientos masivos de erizos (Hernández et al., 2010; Hernández et al., in prep). Este estudio también nos mostró que no todos los sitios funcionaban igual y que sorprendentemente había ciertas regiones del archipiélago donde ese aumento de la temperatura no se traducía en "blooms" de erizos. Para estudiarlo en detalle decidimos comparar qué pasaba en las distintas regiones de Canarias y pudimos demostrar como los erizos sólo aumentaban su densidad en las zonas donde ya había erizos pero no en zonas donde presentaban bajísima densidad, como era el caso de la isla de El Hierro (Oeste del archipiélago). La primera vez que exploramos esta isla nos quedamos maravillados por la gran biomasa de peces y además eran unos peces muy especiales, capaces de depredar activamente sobre un gran espectro de tallas del Diadema (Fig. 6).

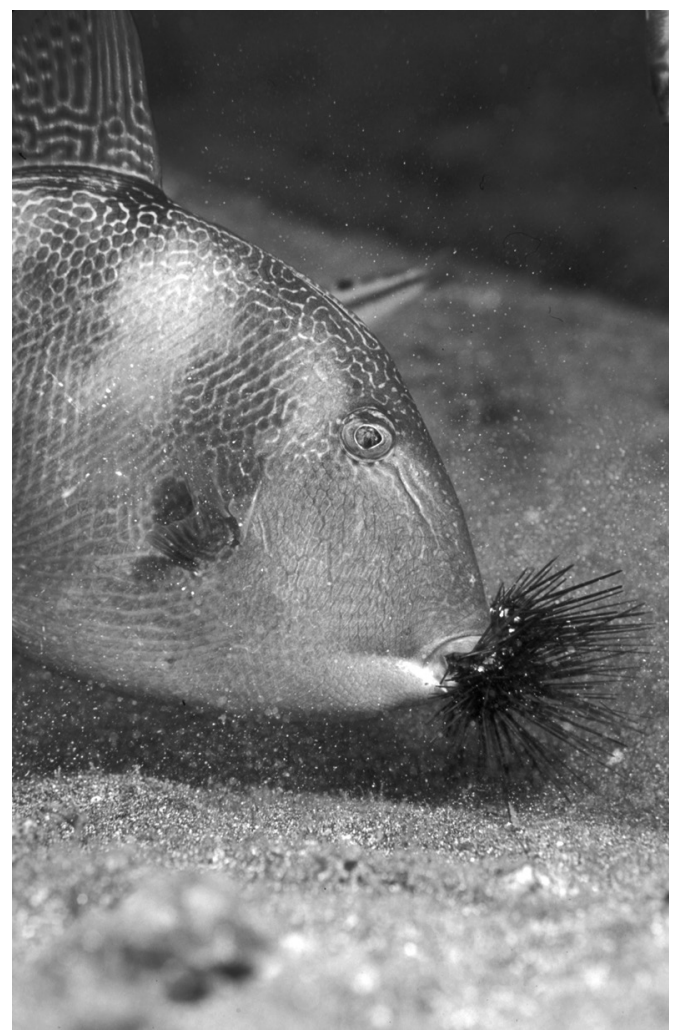

Fig. 6. Balistes capriscus depredando sobre el erizo Diadema africanum. Esta es una imagen que ha sido tomada en la reserva marina del Mar de Las Calmas, isla de El Hierro, Oeste del archipiélago canario.

Fig. 6. Balistes capriscus predating upon the sea urchin Diadema africanum. This picture was taken at the Marine Reserve of Mar de Las Calmas, El Hierro Island, West of the Canarian Arquipelago. 
Después de varios trabajos científicos y una tesis doctoral pudimos definir que existían tres depredadores diurnos (Clemente, 2007; Clemente et al., 2010; 2011) y posiblemente dos más nocturnos (tamboriles y langostas) que no hemos tenido la oportunidad de estudiar en detalle. Concluimos, gracias a estos estudios, que la diversidad específica de depredadores era alta en estos lugares donde los erizos no tienen nada que hacer fuera de sus refugios.

- ¿Cómo frenar la expansión de los blanquizales?

Después de nuestros experimentos en el Oeste del archipiélago comprendimos que las comunidades de algas son resilientes gracias a las altas densidades de depredadores, que son capaces incluso de mitigar los efectos positivos del aumento de las temperaturas en el asentamiento y supervivencia de los erizos. Estos depredadores no solo controlaban a los reclutas sino también a los individuos adultos. Pero quizás lo más sorprendente de todo es que hemos podido comprobar como medidas de conservación y manejo de los ecosistemas como son las reservas marinas son muy útiles para revertir el estado blanquizal y recuperar los bosques de algas, ya que en ellas se reestablece el nivel de los depredadores adecuado (Sangil et al., 2012). Así que la protección resultó en una estrategia más útil que las medidas de eliminación de erizos.

En Canarias habitan dos millones de personas, donde las licencias de pesca han aumentado exponencialmente en los últimos años, además hay una gran cantidad de pescadores ilegales. Son comunes las fotografías donde se puede ver cómo las tallas de las especies han

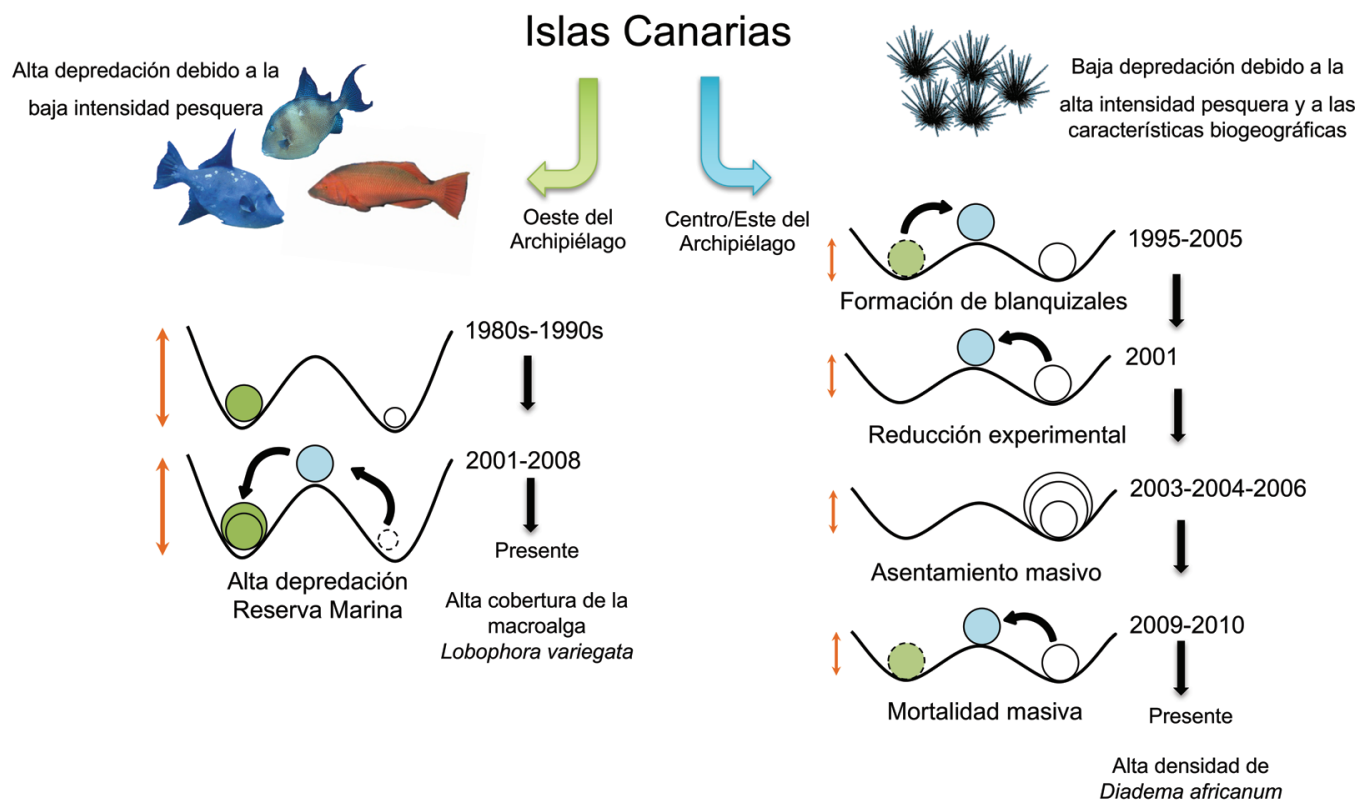

Fig. 7. Diagramas que representan las fases de cambio entre fondos de macroalgas y blanquizales en las islas Canarias. Se han diferenciado las zonas este y oeste del archipiélago por sus diferentes características biogeográficas y de abundancia de depredadores. Para cada región, el diagrama situado en la parte superior del gráfico representa la comunidad más antigua conocida (determinada por referencias bibliográficas), luego, en orden cronológico aparecen las fases de cambio que han sido documentadas, así como los agentes causantes y por último el estado actual de la comunidad.

Fig. 7. Diagrams representing phase shifts between macroalgae beds and sea urchin barrens in the Canary Islands. The east and west zones of the archipelago have been differentiated due to its contrasting biogeographical features and the predator's abundances. For each region, the top diagram (in chronological order) represents the earliest known community assemblage (determine by published references), this is followed by documented phase shifts and associated drivers leading to the actual community state. 
disminuido claramente, incluso en el nivel de los peces herbívoros. Por lo que no es de extrañar que la sobrepesca crónica que han sufrido las Islas haya sido un factor muy relevante en el estado actual de los fondos marinos y en las fluctuaciones de las densidades poblacionales de Diadema. A pesar de esto, quiero acabar de una manera positiva, puesto que las reservas marinas de Canarias han sido un caso claro de éxito, una herramienta que debemos implementar, sobre todo en las islas occidentales (El Hierro y La Palma), ya que gracias a la protección hemos podido recuperar niveles tróficos clave, depredadores de alto nivel trófico, y se ha revertido la situación de blanquizal.

\section{CONCLUSIÓN}

A modo de corolario, hemos podido comprobar como el aumento de las temperaturas genera fluctuaciones de las poblaciones, si bien no se ha revertido la situación de blanquizal sino que en general sus densidades han aumentado. La estrategia de eliminación manual de erizos para la recuperación de los fondos de macroalgas conlleva una erradicación completa, lo que requiere una actividad de mantenimiento permanente por parte de buceadores. Además, la extirpación completa de los erizos, al ser los herbívoros clave puede desencadenar otros problemas de difícil predicción. Por ello, la restauración de la resiliencia del sistema mediante la protección de los depredadores parece ser la estrategia más sensata y con mejores resultados para restablecer y mantener sistemas equilibrados, productivos (Fig. 7). Así pues, no se trata de los erizos y de sus abundancias, o de si los matamos o no, se trata de nosotros mismos, de comprender que para conservar y mantener sistemas saludables debemos dejar de ser tan egoístas con el aprovechamiento de nuestros recursos pesqueros. Tenemos que actuar localmente y unirnos globalmente para promover la creación de reservas marinas y proteger los océanos ¿Lo haremos? Porque la salud en los ecosistemas marinos depende de ello. Así, esta historia ecológica, acaba con el convencimiento de que vale más la pena entender, proteger, amar que matar.

\section{AGRADECIMIENTOS}

En primer lugar, quiero agradecer la invitación del comité de organización del 3er Congreso Latinoamericano de Equinodermos y de manera muy particular a Juan José Alvarado, por haber pensado en mí para dar una charla plenaria, que ha sido el germen del presente trabajo. Agradezco también el esfuerzo de los réferis para encauzar el primer borrador del manuscrito en unos límites más científicos, aunque menos divertidos todo hay que decirlo. Por último, agradezco a todos aquellos que han participado en las distintas expediciones, muestreos, experimentos y reuniones científicas porque han ayudado a que esta historia ecológica se fuera construyendo. Si bien, estos agradecimientos son sobre todo para los que están por venir y van a ayudar a terminarla o por lo menos a que nos acerquemos más a la verdad.

\section{RESUMEN}

Los equinodermos juegan un papel clave en la estructuración de los ecosistemas marinos. A menudo sus densidades poblacionales fluctúan marcadamente y causan cambios en el estado de los ecosistemas que habitan. Los incrementos poblacionales de algunos erizos de mar pueden causar cambios catastróficos en las zonas templadas del planeta, ya que son capaces de eliminar por completo la cobertura de macroalgas erectas. Estos aumentos en las densidades poblacionales resultan en comunidades poco productivas y muy persistentes denominadas blanquizales (ericeras/moradales), o también conocidas por su terminología anglosajona "sea urchin barren grounds". En las zonas templadas las macroalgas son las principales ingenieras del ecosistema y generan un hábitat adecuado para la cría y la alimentación de peces. También son importantes zonas de bio-filtración de aguas costeras y de absorción de CO2. La principal consecuencia de la pérdida de biomasa vegetal es un desequilibrio trófico que en último término puede generar pérdidas económicas importantes para el sector pesquero profesional y turístico. En las zonas tropicales, los blooms de erizos pueden desencadenar fenómenos de bioerosión en las zonas de coral, si bien el fenómeno más importante a destacar ha sido la mortalidad masiva ocurrida en el Caribe en los años 80. En este caso, la desaparición de los erizos favoreció el desarrollo de las comunidades de algas capaces de asfixiar por completo al ecosistema 
de arrecife. Así pues, tanto en zonas templadas como tropicales del planeta, estas fluctuaciones poblacionales de equinoideos generan estados no deseados del ecosistema. En los últimos años, varios trabajos colaborativos a escala global han señalado una clara influencia humana. La actividad humana, mediante la sobrepesca o favoreciendo el calentamiento global, erosiona la resiliencia de los ecosistemas marinos generando estos cambios catastróficos del ecosistema. Para mitigar los efectos de estas fluctuaciones se han utilizado diferentes medidas de manejo. Por ejemplo, en zonas templadas, se han realizado matanzas de erizos (manual o mediante cal viva), extracciones pesqueras o la creación de reservas marinas, con diferente efectividad. En los arrecifes coralinos del Caribe, afectados por la mortalidad de erizos, se han llevado a cabo planes de reintroducción de juveniles, pero con baja efectividad. De todas ellas, la medida ecosistémica más sensata por su carácter preventivo, parece ser la creación de espacios protegidos. Si bien, el impacto del calentamiento global excede las capacidades de manejo de nuestros ecosistemas marinos a nivel local y requerirá de medidas globales más eficientes para prevenir fluctuaciones no deseadas en las poblaciones de erizos.

Palabras clave: erizos de mar; dinámica de poblaciones; ecología de comunidades; cambio climático; depredación; reservas marinas

\section{REFERENCIAS}

Alvarado, J.J., Cortés, J., Guzmán, H., \& Reyes-Bonilla, H. (2016). Bioerosion by the sea urchin Diadema mexicanum along Eastern Tropical Pacific coral reefs. Marine Ecology, 37, 1088-1102. doi:10.1111/ maec. 12372

Alves, F., Chícharo, L.M., Serrao, E., \&Abreu, A.D. (2001). Algal cover and sea urchin spatial distribution at Madeira Island (NE Atlantic). Scientia Marina, 65, 383-392. doi: http://dx.doi.org/10.3989/ scimar.2001.65n4383

Andrew, N.L. \& Underwood, A.J. (1989). Patterns of abundance of the sea urchin Centrostephanus rodgersii (Agassiz) on the central coast of New South Wales, Australia. Journal of Experimental Marine Biology and Ecology, 131, 61-80.

Bak, R.P.M. (1994). Sea urchin bioerosion on coral reefs: place in the carbonate budget and relevant variables. Coral Reefs, 13, 99-104.

Carpenter, R.C. (1988). Mass-mortality of a Caribbean Sea urchin: immediate effects on community metabolism and other herbivores. Proceedings of the National Academy of Science, 85, 511-14.

Carpenter, R.C. (1990). Mass mortality of Diadema antillarum I. Long-term effects on sea urchin population dynamics and coral reef algal communities. Marine Biology 104, 67-77.

Carracedo, J.C., Pérez, F.J., \& Meco, J. (2005). La gea: análisis de una isla en estado post-erosivo de desarrollo. En: Rodríguez, O. (Ed.), Patrimonio natural de la isla de Fuerteventura (pp. 27-44). Cabildo de Fuerteventura-Consejería de Medio Ambiente y Ordenación Territorial del Gobierno de CanariasCentro de la Cultura Popular Canaria. Santa Cruz de Tenerife, Islas Canarias, España.

Carreiro-Silva, M. \& McClanahan, T.R. (2001). Echinoid bioerosion and herbivory on Kenyan corl reefs: role of protection from fishing. Journal of Experimental Marine Biology and Ecology, 262, 133-153.

Clemente, S. (2007). Evolución de las poblaciones del erizo Diadema aff. antillarum en Canarias y valoración de la depredación como factor de control. Tesis doctoral (421 pp.), Universidad de La Laguna, Tenerife, Canary Islands, Spain.

Clemente, S., Hernández, J.C., \& Brito, A. (2009). Evidence of the top-down role of predators in structuring sublittoral rocky-reef communities in a Marine Protected Area and nearby areas of the Canary Islands. ICES Journal of Marine Science, 66, 64-71. doi: https://doi.org/10.1093/icesjms/fsn176

Clemente, S., Hernández, J.C., Rodríguez, A., \& Brito, A. (2010). Identifying keystone predators and the importance of preserving functional diversity in sublittoral rocky bottoms. Marine Ecology Progress Series, 413, 55-67. doi: https://doi.org/10.3354/meps 08700

Clemente, S., Lorenzo-Morales, J., Mendoza, J.C., López, C., Sangil, C., Alves, F., Kaufmann, M. \& Hernández, J.C. (2014). Sea urchin Diadema africanum mass mortality in the subtropical Eastern Atlantic: role of waterborne bacteria in a warming ocean. Marine Ecology Progress Series, 506, 1-14. doi: https://doi. org/10.3354/meps 10829

Dayton, P.K. (1985). Ecology of kelp communities. Annual Review of Ecology and Systematics, 16, 215-245.

Dayton, P.K., Currie, V., Gerrodette, T., Keller, B.D., Rosenthal, R., \& Tresca, D.V. (1984). Patch dynamics and stability of some California kelp communities. Ecological Monograph, 54, 253-289.

Dumont, C., Himmelman, J.H., \& Russell, M.P. (2004). Size-specific movement of green sea urchins Strongylocentrotus droebachiensis on urchin barrens in Eastern Canada. Marine Ecology Progress Series 276, 93-101. doi: 10.3354/meps276093

Dumont, C., Lau D.C.C., Astudillo, J.C., Fong, K.F., Chak, S.T.C., \& Qiub, J.W. (2013). Coral bioerosion by the sea urchin Diadema setosum in Hong Kong: Susceptibility of different coral species. Journal of Experimental Marine Biology and Ecology, 441, 71-79. doi: http://dx.doi.org/10.1016/j.jembe.2013.01.018 
Ebeling, A.W., Laur, D.R., \& Rowley, R.J. (1985). Severe storm disturbances and reversal of community structure in a southern California kelp forest. Marine Biology, 84, 287-294.

Estes, J.A., Tinker, M.T., Williams, T.M., \& Doak, D.F. (1998). Killer Whale predation on sea otters linking oceanic and nearshore ecosystems. Science, 282, 473-476.

Falcón, J.M., Hernández, J.C., Brito, A., García, N., González, G.J., Cruz, A., Herrera, G., Gil-Rodríguez, M.C., \& Clemente, S. (2004). Effects of the sea urchin Diadema antillarum reduction on algae, sessile invertebrates and fish populations in the Canary Islands. Proceedings of the 5th International Symposium on Fauna and Flora of Atlantic Islands FFAIS- 5 Dublin, 24 - 27 August 2004

Fernández-Palacios, J.Mª , de Nascimento, L., Hernández, J.C., Clemente, S., González, A., \& Díaz-González, J.P. (Eds.) (2013) Climate Change Perspectives from the Atlantic: Past, Present and Future. San Cristobal de La Laguna, Tenerife. Servicio de Publicaciones de la Universidad de La Laguna. 729pp.

Filbee-Dexter, K. \& Scheibling, R.E. (2014). Sea urchin barrens as alternative stable states of collapsed kelp ecosystems. Marine Ecology Progress Series, 495, 1-25. doi: $10.3354 / \mathrm{meps} 10573$

Fujita, D. (1998). Strongylocentrotid sea urchin-dominated barren grounds on the Sea of Japan coast of northern Japan. En: Mooi, R. \& Telford, M. (eds.) Echinoderms. (pp 659-664). Balkema, Rotterdam. Holanda.

Glynn, P.W. (1988). El Niño warming, coral mortality and reef framework destruction by echinoid bioerosion in the eastern Pacific. Galaxea, 7, 129-160.

Guidetti, P., Fraschetti, S., Terlizzi, A., \& Boero, F. (2003). Distribution patterns of sea urchins and barrens in shallow Mediterranean rocky reefs impacted by the illegal fishery of the rock-boring mollusc Lithophaga lithophaga. Marine Biology, 143, 1135-1142. doi: 10.1007/s00227-003-1163-Z

Guzmán, H.M. \& Cortes, J. 1989. Coral reef community structure at Caño Island, Pacific Costa Rica. Marine Ecology 10, 23-41.

Hernández, J.C., Clemente, S., Girard, D., Pérez-Ruzafa, A., \& Brito, A. (2010). Effect of temperature on settlement and post -settlement survival in a barrensforming sea urchin. Marine Ecology Progress Series, 413, 69-80. doi: 10.3354/meps08684

Hernández, J.C., Clemente, S., Sangil, C., \& Brito, A. (2008). The key role of the sea urchin Diadema aff. antillarum in controlling macroalgae assemblages throughout the Canary Islands (eastern subtropical Atlantic): a spatio-temporal approach. Marine Environmental Research, 66, 259-270. doi:10.1016/j. marenvres.2008.03.002
Hernández, J.C., Sangil, C., \& Clemente, S. (2013). Sea urchins, natural events and benthic ecosystems functioning in the Canary Islands. En: FernándezPalacios, J.Ma . de Nascimento, L., Hernández, J.C., Clemente, S., González, A. \& Díaz-González, J.P. (Eds.), Climate Change Perspectives from the Atlantic: Past, Present and Future (pp. 487-512). Servicio de Publicaciones de la Universidad de La Laguna, Tenerife, islas Canarias, España.

Hughes, T.P. (1994). Catastrophes, Phase Shifts, and Large-Scale Degradation of a Caribbean Coral Reef. Science, 265,1547-1551.

Jamieson, G.S. \& Campbell, A. (1995). Red sea urchins and kelp in northern British Columbia. En: Skjoldal, H.R., Hopkins, C., Erikstad, K.E., Leinaas, H.P. (eds) Ecology of fjords and coastal waters (pp. 537-547). Elsevier Science, Amsterdam, Holanda.

Johnston, C.S. (1969). Studies on the ecology and primary production of Canary Islands marine algae. Proceedings of the International Seaweed Symposium, 6, 213-222.

Lawrence, J.M. (1975). On the relationship between marine plants and sea urchin. Oceanography and marine biology: an annual review, 13, 213-286.

Lessios, H.A. (1988). Mass mortality of Diadema antillarum in the Caribbean: What Have We Learned? Annual Review of Ecology and Systematics, 19, 371-393.

Lessios, H.A. (2016). The great Diadema antillarum die-off: 30 years later. Annual Review Marine Science, 8, $267-$ 83. doi: 10.1146/annurev-marine-122414-033857

Lessios, H.A., Kessing, B.D., \& Pearse, J.S. (2001). Population structure and speciation in tropical seas, global phylogeography of the sea urchin Diadema. Evolution, 55, 955-975. doi: 10.1111/j.0014-3820.2001. tb00613.x

Ling, S.D., Johnson, C.R., Frusher, S.D., \& Ridgeway, K.R. (2009). Overfishing reduces resilience of kelp beds to climate driven catastrophic phase shift. Proceedings of the National Academy of Science USA, 106, 22341-22345.

Ling, S.D., Scheibling, R.E., Rassweiler, A., Johnson, C.R., Shears, N., Connell, S.D., Salomon, A.K., Norderhaug, K.M., Pérez-Matus, A., Hernández, J.C., Clemente, S. Blamey, L.K., Hereu, B., Ballesteros, E., Sala, E., Garrabou, J., Cebrian, E., Zabala, M., Fujita, D., \& Johnson, L.E. (2014). Global regime shifts dynamics of catastrophic sea urchin overgrazing. Philosophical Transactions of the Royal Society B, 370, 20130269. doi: 10.1098/rstb.2013.0269

López-Yllescas, M. (2012). Relación entre la densidad poblacional de Diadema mexicanum A. Agassiz, 1863 y la cobertura de algas en dos arrecifes coralinos del Pacífico Sur Mexicano, después de un evento de 
mortandad masiva. Tesis de licenciatura. Universidad del Mar, Oaxaca, Méjico. 50p.

Mortensen, T. (1943). A Monograph of the Echinoidea. III. 3. Camarodonta. II. Echinidæ, Strongylocentrotidæ, Parasaleniidæ, Echinometridæ. CA Rietzel, Copenhagen.

Norderhaug, K.M. \& Christie, H.C. (2009). Sea urchin grazing and kelp re-vegetation in the NE Atlantic. Marine Biology Research, 5, 515-528. doi: http:/ dx.doi.org/10.1080/17451000902932985

Rodríguez, A., Hernández, J.C., Clemente, S., \& Coppard, S.E. (2013) A new species of Diadema (Echinode rmata:Echinoidea:Diadematidae) from the eastern Atlantic Ocean and a neotype designation of Diadema antillarum (Philippi, 1845). Zootaxa, 3636(1), 144-170. doi: 10.11646/zootaxa.3636.1.6

Sangil, C., Clemente, S., \& Hernández J.C. (2012). No take areas as an effective tool to restore urchin barrens on subtropical rocky reefs. Estuarine, Coastal and Shelf Science, 112, 207-215. doi: http://dx.doi. org/10.1016/j.ecss.2012.07.025

Sangil, C., Sansón, M., Clemente, S., Afonso-Carrillo, J., \& Hernández, J.C. (2014). Contrasting the species abundance, species density and diversity of seaweed assemblages in alternative states: urchin density as a driver of biotic homogenization. Journal of Sea Research, 85, 92-103. doi: http://dx.doi. org/10.1016/j.seares.2013.10.009

Scheibling, R.E., Hennigar, A.W., \& Balch, T. (1999). Destructive grazing, epiphytism, and disease: the dynamics of sea urchin-kelp interactions in Nova Scotia. Canadian Journal of Fisheries and Aquatic Science, 56, 2300-2314.
Shears, N.T. \& Babcock, R.C. (2003). Continuing trophic cascade effects after 25 years of no-take marine reserve protection. Marine Ecology Progress Series, 246, 1-16.

Steneck, R.S., Vavrinec, J., \& Leland, A.V. (2004). Accelerating trophic-level dysfunction in kelp forests ecosystems of the Western North Atlantic. Ecosystems, 7, 323-332. doi: 10.1007/s10021-004-0240-6

ULL Media (2014). Workshop "Responses of key sea urchin populations to climate change processes: from larvae to ecosystems" [internet]. Disponible en https:// www.youtube.com/playlist?list=PLAqmRmkVzl1OT6tlRqjXD0LgsEGy5pQP

Uthicke, S., Schaffelke, B., \& Byrne, M. (2009). A boombust phylum? Ecological and evolutionary consequences of density variations in echinoderms. Ecological Monographs, 79, 3-24. doi:10.1890/07-2136.1

Vega, J.M.A., Vásquez, J.A., \& Buschmann, A.H. (2005) Population biology of the subtidal kelps Macrocystis integrifolia and Lessonia trabeculata (Laminariales: Phaeophyta) in an upwelling ecosystem of northern Chile: interannual variability and El Niño 1997-98. Revista Chilena de Historia Natural, 78, 33-50. doi: http://dx.doi.org/10.4067/ S0716-078X2005000100004

Wangensteen, O.S., Turon, X., García-Cisneros, A., Recasens, M., Romero, J., \& Palacín, C. (2011). A wolf in sheep's clothing: carnivory in dominant sea urchins in the Mediterranean. Marine Ecology Progress Series, 441, 117-128. doi:10.3354/meps09359

William K.S. (1999). Search for missing otters turns up a few surprises [internet]. New York Times. Disponible en http://www.nytimes.com/1999/01/05/ science/search-for-missing-otters-turns-up-a-fewsurprises.html 